\title{
Risk of severe hematologic toxicities in cancer patients treated with PARP inhibitors: a meta- analysis of randomized controlled trials
}

This article was published in the following Dove Press journal:

Drug Design, Development and Therapy

13 October 2017

Number of times this article has been viewed

\author{
Jian xin Zhou ${ }^{1, *}$ \\ Li jin Feng ${ }^{2, *}$ \\ Xi Zhang ${ }^{3}$
}

'Department of Gynecology, Beijing Obstetrics and Gynecology Hospital, Capital Medical University, Beijing, ${ }^{2}$ Department of Pathology, Shanghai Tenth People's Hospital, Tongji University, School of Medicine, Shanghai, ${ }^{3}$ Department of Radiation Oncology, Affiliated Hospital of Hebei University, Baoding, China

*These authors contributed equally to this work
Correspondence: Xi Zhang Department of Radiation Oncology, Affiliated Hospital of Hebei University, Baoding, 07I000 Hebei, China

Tel $+86312598 \quad I 152$

$\mathrm{Fax}+863125981812$

Email xizhangI225@foxmail.com
Purpose: Hematologic toxicities, including neutropenia, thrombocytopenia, and anemia, are major adverse effects of PARP inhibitors (PARPis), but the incidence rate and overall risk has not been systematically studied. Therefore, we conducted a meta-analysis of published clinical trials to investigate the incidence and relative risks (RRs) of severe (high-grade) hematologic events in cancer patients treated with PARPis.

Methods: PubMed, Embase, and oncology conference proceedings were searched for relevant studies. Eligible studies were Phase II and III randomized controlled trials (RCTs) of PARPis in cancer patients with adequate safety data on hematologic toxicities. The summary incidence, RRs, and 95\% confidence intervals (CIs) were calculated.

Results: A total of 2,479 patients from 12 RCTs revealed that the incidence of PARPiassociated severe hematologic toxicities was, respectively: neutropenia: 32.9\% (95\% CI, 20.5\%-48.3\%); thrombocytopenia: $15.9 \%$ (95\% CI, 9.5\%-25.4\%), and anemia: $9.1 \%$ (95\% CI, $5.1 \%-15.7 \%$ ). Olaparib was associated with an increased risk of severe neutropenia. Veliparib was associated with an increased risk of severe neutropenia and thrombocytopenia. Niraparib was associated with an increased risk of severe thrombocytopenia, anemia, and neutropenia. When stratified by combination therapy, significantly increased risk of hematologic toxicities was observed for patients treated with PARPis monotherapy and PARPis combined with single-agent chemotherapy.

Conclusion: Treatment with PARPis olaparib, veliparib, and niraparib is associated with a significant increase in the risk of hematologic toxicities in cancer patients, and frequent clinical monitoring should be emphasized when managing these PARPis.

Keywords: hematologic toxicities, PARP inhibitors, cancer, meta-analysis, RCTs

\section{Introduction}

PARPs are a family of nuclear enzymes, which functions in DNA repair, cell proliferation, differentiation, and transformation. ${ }^{1}$ PARP-1, PARP-2, and PARP-3 have shown activity specific to DNA repair and genomic stability. ${ }^{2}$ PARP-1 is the best described member of this family and plays a critical role in the repair of singlestrand breaks (SSB) through the base excision repair (BER) pathway. Inhibition of PARP-1 and its DNA repair mechanisms can result in persistence of single-strand DNA breaks which eventually lead to formation of double-strand breaks. However, cells deficient in BRCA1 and 2 are unable to fully utilize homologous recombination to repair these double-strand breaks, then a low-fidelity repair by non-homologous end joining is activated, and this absence of an accurate repair mechanism results in cell death..$^{3,4}$ Olaparib (Lynparza ${ }^{\mathrm{TM}}$; AstraZeneca, London, UK) was the first PARP 
inhibitor (PARPi) to gain US Food and Drug Administration (FDA) approval, with activity in deleterious germline mutation of BRCA1 or BRCA2 and advanced ovarian cancer in patients who have received three or more prior chemotherapy regimens. ${ }^{5}$ Then, the FDA granted accelerated approval to rucaparib for the treatment of patients with deleterious BRCA mutation (germline and/or somatic) associated advanced ovarian cancer who have been treated with two or more chemotherapies. ${ }^{6}$ Niraparib (ZEJULA ${ }^{\text {TM}}$; Tesaro, Waltham, MA, USA) is another PARPi, which has been recently approved for the maintenance treatment of adult patients with recurrent epithelial ovarian, fallopian tube, or primary peritoneal cancer who have achieved complete or partial response to platinum-based chemotherapy. ${ }^{7}$ Multiple other PARPis, including veliparib and talazoparib, are currently being evaluated in many clinical trials. ${ }^{8}$ As the use of those agents is expected to increase in the near future, an appreciation of their toxicity profiles is therefore urgently needed. PARPis have been accompanied by a specific pattern of toxicities including fatigue, gastrointestinal symptoms (nausea, vomiting, diarrhea, and constipation). ${ }^{9-11}$ Additionally, hematologic toxicities are also described to be frequent adverse events (AEs) for PARPis, especially combined with chemotherapy. Life-threatening events (severe infection, bleeding) might occur if decreased blood cells have not been managed in a timely manner. However, the incidence of severe hematologic toxicities with PARPis monotherapy or PARPis with chemotherapy vary widely across clinical trials. Therefore, a formal assessment of the incidence and relative risks (RRs) of hematologic toxicities associated with PARPis is needed. This meta-analysis was conducted to quantify the overall incidence and RRs of severe hematologic toxicities among cancer patients treated with PARPis.

\section{Methods}

\section{Data sources and search strategy}

This meta-analysis was performed in accordance with the PRISMA (Preferred Reporting Items for Systemic Reviews and Meta-Analyses) recommendations. ${ }^{12}$ An independent review of citations from PubMed and Embase from January 2000 to May 2017 was conducted. Abstracts presented at the annual meetings of the American Society of Clinical Oncology and the European Society of Medical Oncology were also searched to identify relevant clinical trials. Additionally, we searched the clinical trial registration website (http://www. ClinicalTrials.gov) to obtain information on registered prospective trials. Keywords were olaparib, niraparib, veliparib, rucaparib, talazoparib, PARP inhibitors, RCT, and cancer.
The search was restricted to randomized controlled trials (RCTs) published in English. When duplicate publications were identified, only the most complete, recent, and updated report of clinical trials was included in the meta-analysis.

\section{Study selection}

Clinical trials meeting the following criteria were included: 1) prospective randomized controlled Phase II and III clinical trails in cancer patients, 2) participants assigned to treatment with PARPis or control (placebo, chemotherapy or radiotherapy), 3) available data regarding events or event rates and sample size of hematologic toxicities including neutropenia, thrombocytopenia, and anemia. Phase I and single-arm Phase II trials were excluded due to lack of control groups.

\section{Data extraction and clinical end points}

Data extraction was independently conducted by two investigators, and any discrepancies were resolved by consensus. For each study, the following information was extracted: first author's name, year of publication, underlying malignancy, trial phase, number of enrolled subjects for analysis, dosage of the PARPis, and the dosing schedules used in treatment arms and control arms. Study quality of RCTs was assessed according to the Jadad scale including randomization, blinding, and withdrawals. ${ }^{13}$ Severe (high-grade, grade 3 or 4 ) hematologic toxicities were defined according to the third or fourth version of the Common Terminology Criteria for Adverse Events (CTCAE) of the National Cancer Institute.

\section{Statistical analysis}

The principal summary measures were incidence, RRs, and corresponding 95\% confidence intervals (CIs) of severe hematologic adverse events (AEs). The number of patients experiencing severe hematologic AEs and the total number of patients receiving the study drug were extracted from the safety profiles of all selected studies. Studies that had a comparative arm were used to calculate RRs of severe hematologic toxicities in patients assigned to PARPis versus placebo/controls in the same trial. For the meta-analysis, both the fixed effects model (weighted with inverse variance) and the random effects model were considered. ${ }^{14,15}$ The $\chi^{2}$ based $Q$ statistic was applied to estimate between-study heterogeneity, and inconsistency was quantified with the $I^{2}$ statistic, which represents the percentage of total variation across studies that is attributable to heterogeneity rather than chance. ${ }^{16}$ Heterogeneity was considered statistically significant when $P<0.1$. When substantial heterogeneity was 
not observed, the pooled estimate was calculated based on the fixed effects model. If substantial heterogeneity existed, data were analyzed using a random effects model. Publication bias was evaluated by the Begg and the Egger test. ${ }^{17,18}$ A two-tailed $P$-value $<0.05$ was considered statistically significant. All statistical analyses were performed using version 2 of the Comprehensive Meta Analysis program (Biostat, Englewood, NJ, USA).

\section{Results}

\section{Search results}

Our search identified a total of 431 potentially relevant articles. The detailed selection process and reasons for exclusion are presented in Figure 1. After the selection process, a total of 12 RCTs were considered eligible for the meta-analysis including eleven Phase II trials and 1 Phase III trial.

\section{Study characteristics}

In total, 2,479 patients from 12 RCTs were eligible for the meta-analysis and they had a variety of cancers: ovarian cancer ${ }^{9,10,20-22}$ (five trials), non small-cell lung cancer ${ }^{23,25}$ (two trials), breast cancer ${ }^{24,27}$ (two trials), melanoma ${ }^{11}$ (one trial), gastric cancer ${ }^{19}$ (one trial), and small-cell lung cancer ${ }^{26}$ (one trial). Of the included studies, four trials evaluated olaparib ${ }^{10,19,20,22}$ and seven trials evaluated veliparib, ${ }^{11,21,23-27}$ whereas one trial evaluated niraparib. ${ }^{9}$ For the 12 included studies, ten studies reported safety data on anemia, ${ }^{9-11,19-25}$ nine studies reported safety data on neutropenia, ${ }^{9-11,19,21,24-27}$ and nine studies reported safety data

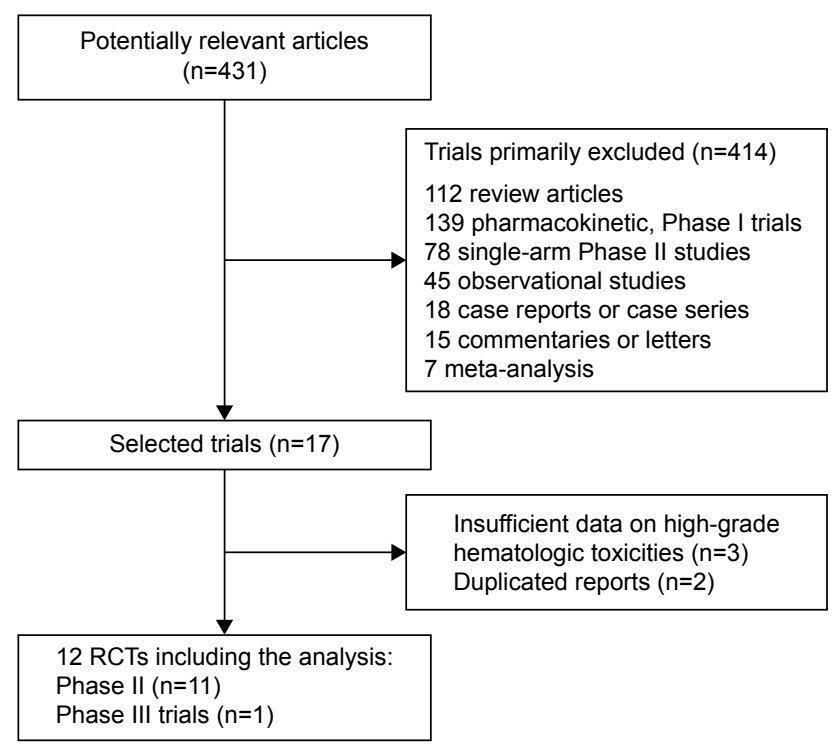

Figure I Flow chart of selection process for trials included in meta-analysis. Abbreviation: RCT, randomized controlled trial. on thrombocytopenia. ${ }^{9-11,21,23-27}$ The mean Jadad score was 4.2 (range, 3-5) indicating the overall methodological quality of the included studies was generally good and fair. Jadad scores are listed for each trial in Table 1. All 12 trials reported hematologic AEs according to the National Cancer Institute's CTCAE version three or four criteria. All selected trials included patients with an Eastern Cooperative Oncology Group performance status of two or less and with adequate organ, coagulation, and hematological functions.

\section{Overall incidence of severe hematologic toxicities}

For the incidence of severe hematologic toxicities, all PARPis treatment arms were considered. The summary incidences of severe neutropenia (Figure 2A), thrombocytopenia (Figure 2B), and anemia (Figure 2C) in patients receiving PARPis were $32.9 \%$ (95\% CI, 20.5\%-48.3\%), $15.9 \%$ (95\% CI, 9.5\%-25.4\%), and 9.1\% (95\% CI, 5.1\%-15.7\%), respectively. The test for heterogeneity was significant for severe neutropenia, thrombocytopenia, and anemia, and random effects model was used. We further conducted subgroup analysis to evaluate the incidence of hematologic toxicities based on each drug. Thrombocytopenia and anemia were the most common hematologic toxicity events with niraparib, and olaparib resulted in higher incidence of neutropenia when compared with niraparib and veliparib. There were significant differences among the incidences of neutropenia $(P<0.001)$, thrombocytopenia $(P<0.001)$, and anemia $(P<0.001)$ induced by each PARPi. When stratified by PARPi-based regimens (PARP monotherapy versus PARPis combined with single-agent chemotherapy versus PARPis combined with carboplatin and paclitaxel), the difference was significant for neutropenia $(P=0.030)$, which registered higher incidence in PARPis combined with carboplatin and paclitaxel group (Table 2).

\section{RRs of severe hematologic toxicities}

The RRs of hematologic toxicities were calculated by comparing only with those assigned to a control treatment in the same trial. The RRs of severe neutropenia (Figure 3A), thrombocytopenia (Figure 3B), and anemia (Figure 3C) were 2.11 (95\% CI, 1.31-3.40; $P=0.002), 2.42$ (95\% CI, 1.2374.76; $P=0.011)$, and $2.27(95 \% \mathrm{CI}, 1.08-4.81 ; P=0.032)$, respectively. The test for heterogeneity was significant for neutropenia (heterogeneity test: $I^{2}=79.83 \%, P<0.001$ ), thrombocytopenia (heterogeneity test: $I^{2}=71.06 \%, P=0.001$ ), and anemia (heterogeneity test: $I^{2}=52.61 \%, P=0.025$ ), and random effects model was used. 
Table I Baseline characteristics of 12 randomized controlled trials in the meta-analysis

\begin{tabular}{|c|c|c|c|c|c|c|c|c|}
\hline Study & Phase & $\begin{array}{l}\text { Underlying } \\
\text { malignancy }\end{array}$ & Treatment arm & Control arm & $\begin{array}{l}\text { Treatment } \\
\text { arm }\end{array}$ & $\begin{array}{l}\text { Control } \\
\text { arm }\end{array}$ & $\begin{array}{l}\text { CTCAE } \\
\text { version }\end{array}$ & $\begin{array}{l}\text { Jadad } \\
\text { score }\end{array}$ \\
\hline Oza et al, $2015^{10}$ & II & OC & Olaparib 200 mg twice daily plus PC & PC & 81 & 75 & 3.0 & 3 \\
\hline \multirow[t]{2}{*}{ Kaye et al, $2012^{22}$} & II & OC & Olaparib 200 mg twice daily & PLD & 32 & 32 & 3.0 & 3 \\
\hline & & & Olaparib 400 mg twice daily & PLD & 32 & & & \\
\hline Bang et al, $2015^{19}$ & II & $\begin{array}{l}\text { Gastric } \\
\text { cancer }\end{array}$ & $\begin{array}{l}\text { Olaparib } 100 \mathrm{mg} \text { twice daily plus } \\
\text { paclitaxel }\end{array}$ & Placebo/paclitaxel & 61 & 62 & 3.0 & 5 \\
\hline $\begin{array}{l}\text { Ledermann et al, } \\
2012^{20}\end{array}$ & II & OC & Olaparib 400 mg twice daily & Placebo & 136 & 128 & 3.0 & 5 \\
\hline Mirza et al, $2016^{9}$ & III & OC & Niraparib 300 mg once daily & Placebo & 367 & 179 & 4.0 & 5 \\
\hline $\begin{array}{l}\text { Kummar et al, } \\
2015^{21}\end{array}$ & II & OC & $\begin{array}{l}\text { Veliparib } 60 \mathrm{mg} \text { once daily plus oral } \\
\text { cyclophosphamide }\end{array}$ & $\begin{array}{l}\text { Oral } \\
\text { cyclophosphamide }\end{array}$ & 37 & 38 & 4.0 & 3 \\
\hline $\begin{array}{l}\text { Middleton et al, } \\
2015^{\prime \prime}\end{array}$ & II & Melanoma & $\begin{array}{l}\text { Veliparib } 20 \mathrm{mg} \text { twice daily plus TMZ } \\
\text { Veliparib } 40 \mathrm{mg} \text { twice daily plus TMZ }\end{array}$ & Placebo plus TMZ & $\begin{array}{l}116 \\
115\end{array}$ & 113 & 3.0 & 5 \\
\hline $\begin{array}{l}\text { Ramalingam et al, } \\
2017^{25}\end{array}$ & II & NSCLC & Veliparib I20 mg twice daily plus PC & PC & 105 & 52 & 4.0 & 5 \\
\hline Rugo et al, $2016^{24}$ & II & $\mathrm{BC}$ & Veliparib 50 mg twice daily plus PC & Paclitaxel & 72 & 44 & & 3 \\
\hline Chabot et al, $2017^{23}$ & II & NSCLC & $\begin{array}{l}\text { Veliparib } 50 \text { mg twice daily plus WBRT } \\
\text { Veliparib } 200 \text { mg twice daily plus WBRT }\end{array}$ & Placebo plus WBRT & $\begin{array}{l}103 \\
102\end{array}$ & 101 & 4.0 & 5 \\
\hline $\begin{array}{l}\text { Pietanza et al, } \\
2016^{26}\end{array}$ & II & SCLC & Veliparib 40 mg twice daily plus TMZ & TMZ plus placebo & 50 & 50 & 4.0 & 4 \\
\hline Han et al, $2017^{27}$ & II & BC & Veliparib $120 \mathrm{mg}$ twice daily plus PC & PC & 97 & 99 & 4.0 & 4 \\
\hline
\end{tabular}

Abbreviations: OC, ovarian cancer; NSCLC, non-small cell lung cancer; BC, breast cancer; PLD, pegylated liposomal doxorubicin; PC, paclitaxel and carboplatin; WBRT, whole-brain radiation therapy; TMZ, temozolomide.

\section{RRs of severe hematologic toxicities by PARPis}

In order to explore the impact of different PARPis on the RRs of hematologic toxicities, we calculated RRs according to drug type (Table 3). In the niraparib trial, a significantly increased risk was observed for neutropenia with an RR of 11.71 (95\% CI, 3.74-36.64, $P<0.001$ ), thrombocytopenia with an RR of $60.48(95 \% \mathrm{CI}, 8.52-429.25, P<0.001)$ and anemia with an RR of 91.47 (95\% CI, 5.71-1464.79, $P=0.001)$. As for veliparib as the study drug, the RR of neutropenia was 2.33 (95\% CI, 1.09-5.01, $P=0.020$ ), the RR of thrombocytopenia was $1.97(95 \% \mathrm{CI}, 1.11-3.48, P=0.020)$, and the RR of anemia was 1.44 (95\% CI, 0.74-2.80, $P=0.278)$. For olaparib treated patients, we observed an RR of neutropenia of 1.34 ( $95 \% \mathrm{CI}, 1.02-1.77, P=0.037)$, an RR of thrombocytopenia of 0.77 (95\% CI, 0.25-2.42, $P=0.657)$, and an RR of anemia of 1.50 (95\% CI, 0.77-2.95, $P=0.236$ ). Significant differences in RRs were detected for anemia ( $P=0.021)$, neutropenia $(P=0.001)$, and thrombocytopenia $(P=0.001)$.

\section{RRs of severe hematologic toxicities by PARPi-based regimens}

We also carried out a subgroup analysis according to PARPibased regimens (Table 3). Concerning the RRs of PARPis monotherapy (only one trial evaluated neutropenia and thrombocytopenia), ${ }^{9}$ we observed an RR of neutropenia of 11.71 (95\% CI, 3.74-36.64, $P<0.001$ ), an RR of thrombocytopenia of 60.48 ( $95 \%$ CI, 8.52-429.25, $P<0.001)$, and an RR of anemia of 13.36 (95\% CI, 3.17-56.19, $P<0.001)$. As for PARPis combined with single-agent chemotherapy group, we observed an RR of neutropenia of 1.82 (95\% CI, 1.31-2.53, $P<0.001)$, an RR of thrombocytopenia of 2.78 (95\% CI, 1.06-7.27, $P=0.038)$, and an RR of anemia of $1.42(95 \%$ CI, 0.67-3.01, $P=0.354)$. With regard to PARPis combined with carboplatin and paclitaxel, we observed an RR of neutropenia of 1.29 ( $95 \%$ CI, $0.75-2.22, P=0.361)$, an RR of thrombocytopenia of 1.15 (95\% CI, $0.78-1.71, P=0.483)$, and an RR of anemia of 1.45 ( $95 \%$ CI, 0.71-2.96, $P=0.314)$. Significant differences in RRs were detected for neutropenia $(P=0.003)$, thrombocytopenia $(P=0.001)$, and anemia $(P=0.045)$.

\section{RRs of severe hematologic toxicities related to control therapy}

The risk of severe hematologic toxicities might be related to control therapy (Table 3). In the studies with placebo as the control arm (only one trial evaluated neutropenia and thrombocytopenia), ${ }^{9}$ the RRs were 11.71 for neutropenia (95\% CI, 3.74-36.64, $P<0.001), 60.48$ for thrombocytopenia (95\% CI, 8.52-429.25, $P<0.001$ ), and 17.00 for anemia (95\% CI, 3.22-89.82, $P=0.001)$. In the studies with singleagent chemotherapy as the control arm, the RRs were 3.52 
A

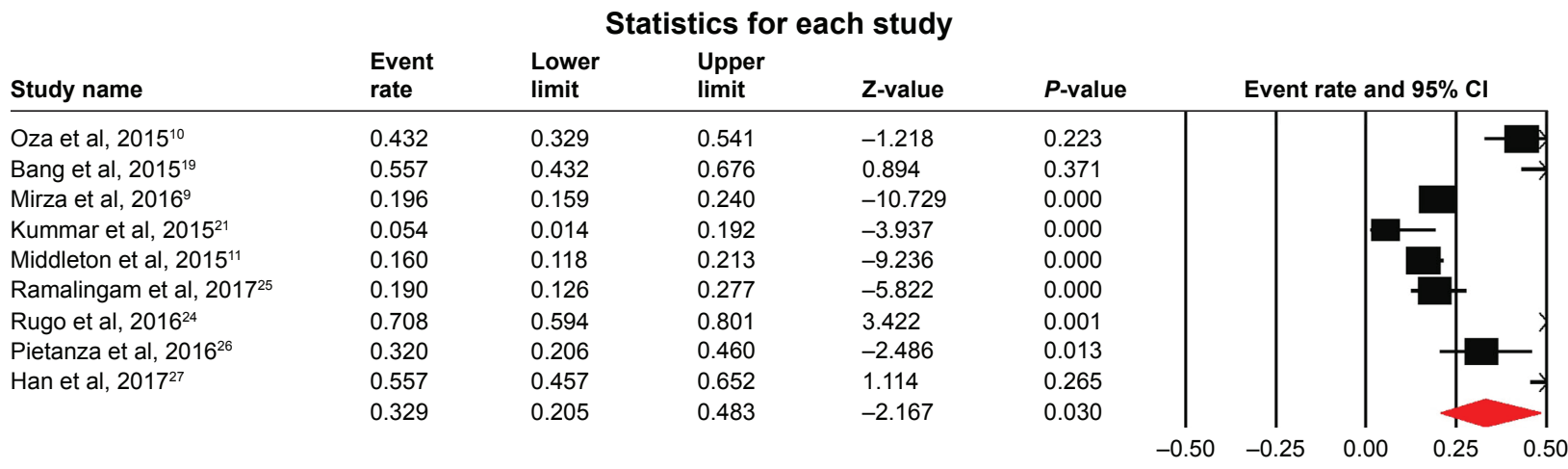

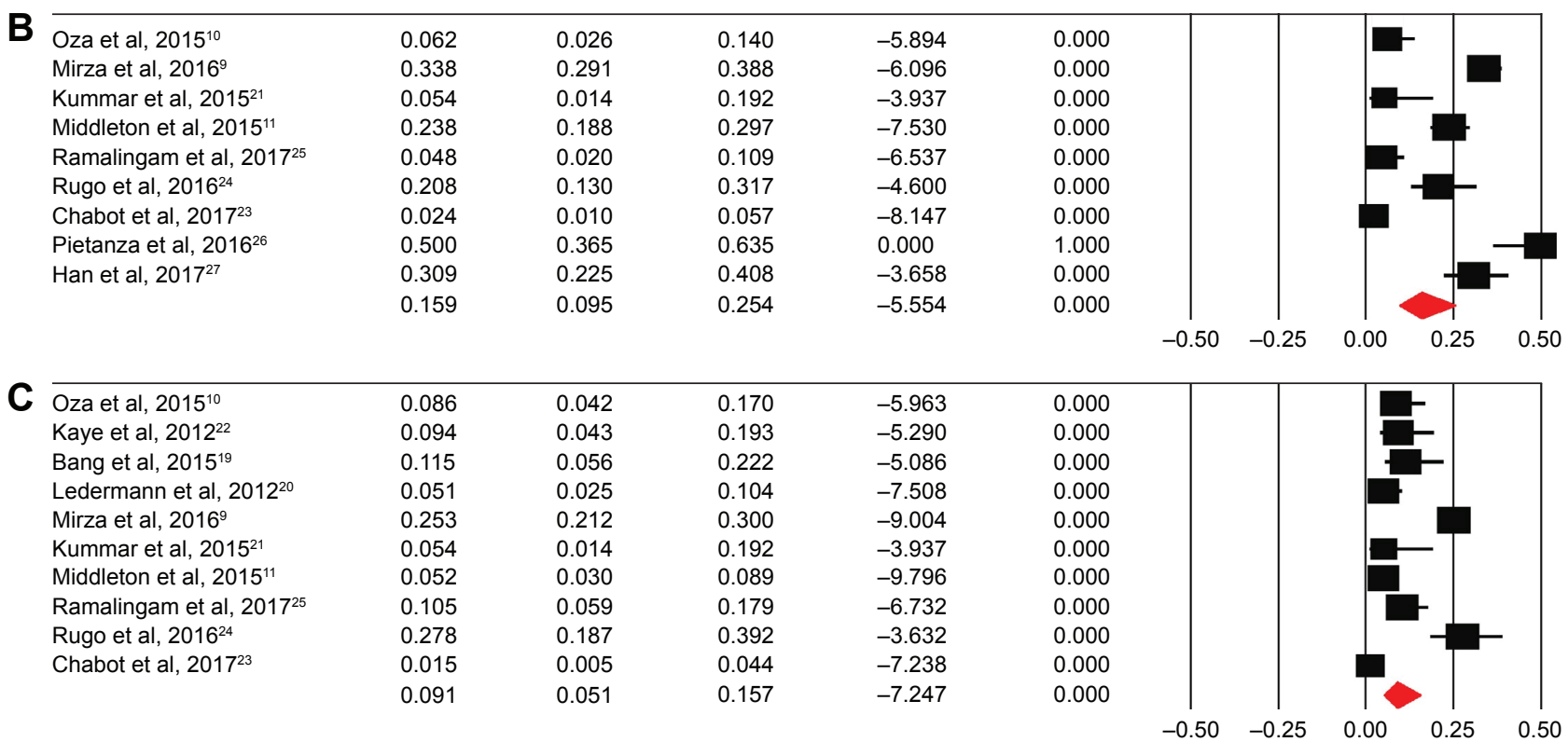

Figure 2 Forest plot for meta-analysis of incidence of severe hematologic toxicities: neutropenia (A), thrombocytopenia (B), and anemia (C).

for neutropenia $(95 \% \mathrm{CI}, 1.49-8.31, P=0.004), 3.44$ for thrombocytopenia (95\% CI, 1.27-9.33, $P=0.015)$, and 1.88 for anemia (95\% CI, 0.93-3.78, $P=0.078$ ). In the studies with carboplatin and paclitaxel as the control arm, the RRs were 1.05 for neutropenia ( $95 \% \mathrm{CI}, 0.86-1.29, P=0.626)$, 1.09 for thrombocytopenia ( $95 \% \mathrm{CI}, 0.73-1.62, P=0.679$ ) and 1.18 for anemia (95\% CI, 0.56-2.48, $P=0.664)$. Significant differences in RRs were detected for neutropenia

Table 2 Incidence of severe hematologic toxicities stratified by drug type and combination therapy

\begin{tabular}{|c|c|c|c|c|c|c|c|}
\hline & \multicolumn{2}{|l|}{ Olaparib } & \multicolumn{2}{|l|}{ Niraparib } & \multicolumn{2}{|l|}{ Veliparib } & \multirow[t]{3}{*}{ P difference ${ }^{\#}$} \\
\hline & \multicolumn{2}{|c|}{ Incidence $(95 \% \mathrm{Cl})$} & \multicolumn{2}{|c|}{ Incidence $(95 \% \mathrm{Cl})$} & \multicolumn{2}{|c|}{ Incidence $(95 \% \mathrm{Cl})$} & \\
\hline & PARPis & Control & PARPis & Control & PARPis & Control & \\
\hline Neutropenia & $49.1(37.0-61.2)$ & $36.5(28.9-44.9)$ & $19.6(15.9-24.0)$ & $1.7(0.5-5.1)$ & $29.9(\mid 4.2-52.5)$ & $10.3(2.8-3||)$. & $<0.001$ \\
\hline Thrombocytopenia & $6.2(2.6-14.0)$ & $8.0(3.6-16.7)$ & $33.8(29.1-38.8)$ & $0.6(0.1-3.9)$ & I 5.1 (7.7-27.7) & $10.5(5.1-20.1)$ & $<0.001$ \\
\hline \multirow[t]{4}{*}{ Anemia } & $8.2(5.7-1 \mathrm{I} .8)$ & $4.7(1.7-12.8)$ & $25.3(21.2-30.0)$ & $0.3(0-4.3)$ & $7.3(2.7-18.4)$ & $4.9(2.6-9.2)$ & $<0.001$ \\
\hline & \multicolumn{2}{|c|}{ PARPis monotherapy } & \multicolumn{2}{|c|}{ PARPis with single-agent CT } & \multicolumn{2}{|c|}{ PARPis with PC } & $P$ difference \\
\hline & \multicolumn{2}{|c|}{ Incidence $(95 \% \mathrm{Cl})$} & \multicolumn{2}{|c|}{ Incidence $(95 \% \mathrm{Cl})$} & \multicolumn{2}{|c|}{ Incidence $(95 \% \mathrm{Cl})$} & \\
\hline & PARPis & Control & PARPis & Control & PARPis & Control & \\
\hline Neutropenia & $19.6(15.9-24.0)$ & $1.7(0.5-5.1)$ & $24.1(9.6-48.8)$ & $9.2(2.0-33.6)$ & $46.2(25.9-67.9)$ & $27.8(13.2-49.4)$ & 0.030 \\
\hline Thrombocytopenia & $33.8(29.1-38.8)$ & $0.6(0.1-3.9)$ & $24.0(9.4-49.2)$ & $12.9(8.7-18.8)$ & | 3.0 (5.2-28.9) & $8.9(2.8-24.8)$ & 0.048 \\
\hline Anemia & $1 \mid .4(3.6-3 \mid .0)$ & $0.7(0.2-2.8)$ & $7.0(3.9-12.0)$ & $4.6(1.2-15.8)$ & $14.3(6.3-29.4)$ & $7.3(4.0-12.8)$ & 0.324 \\
\hline
\end{tabular}

Note: \#Difference in the incidence of different PARPis and combination therapy.

Abbreviations: PARPis, PARP inhibitors; PC, carboplatin and paclitaxel; CT, chemotherapy. 
A

\begin{tabular}{|c|c|c|c|c|c|c|c|c|c|}
\hline \multirow[b]{2}{*}{ Study name } & \multicolumn{5}{|c|}{ Statistics for each study } & \multicolumn{4}{|c|}{ Events/total } \\
\hline & $\begin{array}{l}\text { Risk } \\
\text { ratio }\end{array}$ & $\begin{array}{l}\text { Lower } \\
\text { limit }\end{array}$ & $\begin{array}{l}\text { Upper } \\
\text { limit }\end{array}$ & Z-value & $P$-value & Group-A & Group-B & Risk ratio and $95 \% \mathrm{Cl}$ & \\
\hline Oza et al, $2015^{10}$ & 1.246 & 0.837 & 1.857 & 1.083 & 0.279 & $35 / 81$ & $26 / 75$ & & \\
\hline Bang et al, $2015^{19}$ & 1.440 & 0.980 & 2.116 & 1.857 & 0.063 & $34 / 61$ & $24 / 62$ & & \\
\hline Mirza et al, $2016^{9}$ & 11.706 & 3.740 & 36.638 & 4.226 & 0.000 & $72 / 367$ & $3 / 179$ & & \\
\hline Kummar et al, $2015^{21}$ & 5.132 & 0.255 & 103.413 & 1.067 & 0.286 & $2 / 37$ & $0 / 38$ & & \\
\hline Middleton et al, $2015^{11}$ & 3.017 & 1.312 & 6.937 & 2.599 & 0.009 & $37 / 231$ & $6 / 113$ & & \\
\hline Ramalingam et al, $2017^{25}$ & 0.825 & 0.438 & 1.556 & -0.593 & 0.553 & $20 / 105$ & $12 / 52$ & & \\
\hline Rugo et al, $2016^{24}$ & 31.167 & 4.464 & 217.582 & 3.469 & 0.001 & $51 / 72$ & $1 / 44$ & & \\
\hline Pietanza et al, $2016^{26}$ & 4.000 & 1.438 & 11.128 & 2.656 & 0.008 & $16 / 50$ & $4 / 50$ & & \\
\hline \multirow[t]{2}{*}{ Han et al, $2017^{27}$} & 1.021 & 0.793 & 1.314 & 0.158 & 0.874 & $54 / 97$ & $54 / 99$ & & \\
\hline & 2.110 & 1.308 & 3.403 & 3.063 & 0.002 & $321 / 1,101$ & $130 / 712$ & & \\
\hline
\end{tabular}

B

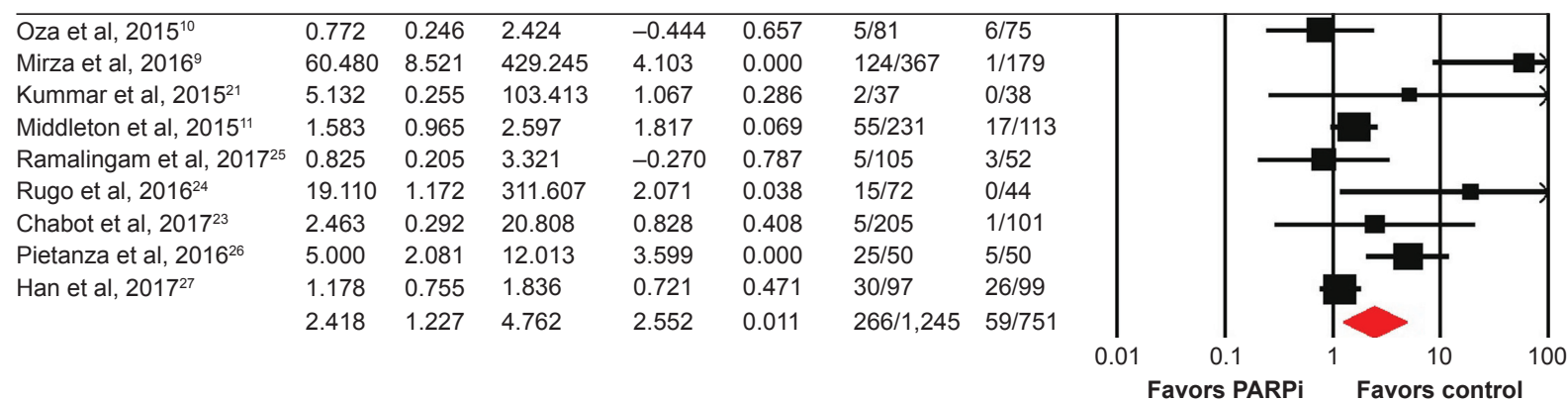

C

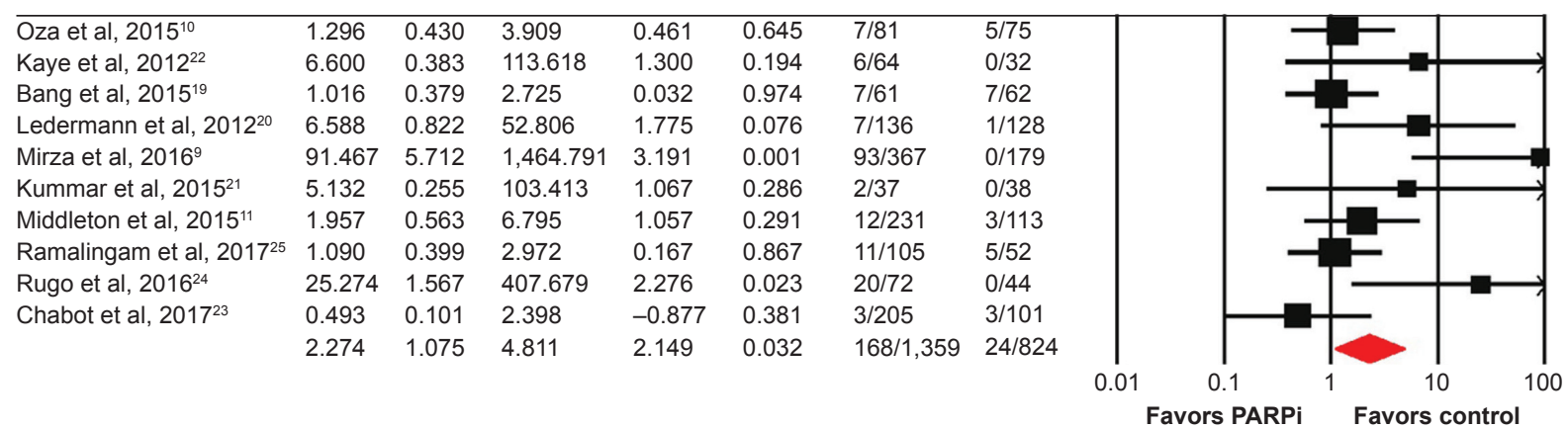

Figure 3 Forest plot for meta-analysis of relative risk of severe hematologic toxicities: neutropenia (A), thrombocytopenia (B), and anemia (C).

Abbreviation: PARPi, PARP inhibitor.

Table 3 Relative risk of hematologic toxicities with PARPis according to drug type, combination therapy, and controlled therapy

\begin{tabular}{|c|c|c|c|c|}
\hline \multirow[t]{2}{*}{ Drug type } & \multirow{2}{*}{$\frac{\text { Olaparib }}{\text { RR }(95 \% \mathrm{Cl})}$} & \multirow{2}{*}{$\frac{\text { Niraparib }}{\text { RR }(95 \% \mathrm{CI})}$} & \multirow{2}{*}{$\frac{\text { Veliparib }}{\text { RR }(95 \% \mathrm{CI})}$} & \multirow[t]{2}{*}{$P$ difference } \\
\hline & & & & \\
\hline Neutropenia & $1.34(1.02-1.77)$ & II.7I (3.74-36.64) & $2.33(1.09-5.01)$ & 0.001 \\
\hline Thrombocytopenia & $0.77(0.25-2.42)$ & $60.48(8.52-429.25)$ & $1.97(1.11-3.48)$ & 0.001 \\
\hline Anemia & $1.50(0.77-2.95)$ & $91.47(5.7 \mathrm{I}-\mathrm{I}, 464.79)$ & $1.44(0.74-2.80)$ & 0.021 \\
\hline \multirow[t]{2}{*}{ Combination therapy } & PARPis monotherapy & PARPis with single-agent CT & PARPis with PC & $P$ difference ${ }^{\#}$ \\
\hline & $\overline{R R}(95 \% \mathrm{Cl})$ & $\mathbf{R R}(95 \% \mathrm{Cl})$ & $\overline{\operatorname{RR}(95 \% \mathrm{CI})}$ & \\
\hline Neutropenia & II.7I (3.74-36.64) & $1.82(1.3 \mathrm{I}-2.53)$ & $1.29(0.75-2.22)$ & 0.003 \\
\hline Thrombocytopenia & $60.48(8.52-429.25)$ & $2.78(1.06-7.27)$ & $1.15(0.78-1.7 I)$ & 0.001 \\
\hline Anemia & $13.36(3.17-56.19)$ & $1.42(0.67-3.01)$ & $\mathrm{I} .45(0.7 \mathrm{I}-2.96)$ & 0.045 \\
\hline \multirow[t]{2}{*}{ Controlled therapy } & Placebo & Single-agent CT & PC & $P$ difference ${ }^{\#}$ \\
\hline & $\overline{R R}(95 \% \mathrm{CI})$ & RR (95\% Cl) & RR (95\% CI) & \\
\hline Neutropenia & II.7I (3.74-36.64) & $3.52(|.49-8.3|)$ & I.05 (0.86-I.29) & $<0.001$ \\
\hline Thrombocytopenia & $60.48(8.52-429.25)$ & $3.44(1.27-9.33)$ & $1.09(0.73-1.62)$ & $<0.001$ \\
\hline Anemia & $17.00(3.22-89.82)$ & $1.88(0.93-3.78)$ & $1.18(0.56-2.48)$ & 0.076 \\
\hline
\end{tabular}

Note: difference in the RR of different PARPis, combination therapy and controlled therapy.

Abbreviations: PARPis, PARP inhibitors; RR, relative risk; CT, chemotherapy; PC, carboplatin and paclitaxel. 
$(P<0.001)$ and thrombocytopenia $(P<0.001)$, but not for anemia $(P=0.076)$.

\section{Publication bias}

We found no evidence of publication bias for RRs of severe thrombocytopenia by either the Egger or the Begg test. For RRs of severe anemia, the Egger test suggested some evidence of publication bias, however, the Begg test showed no evidence of bias. Moreover, evidence of publication bias was observed for severe neutropenia by both the Egger and the Begg test.

\section{Discussion}

Toxicities of PARPis appear to be similar to cytotoxic chemotherapeutic agents. Data from previous studies have shown that the most common grade 3-4 toxicities of PARPis were nausea, vomiting, and hematological toxicities, leading to dose delays and interruptions. ${ }^{28,29}$ Our meta-analysis was able to demonstrate that treatment with PARPis was associated with a significantly increased risk of developing hematologic toxicities in cancer patients. As for olaparib and veliparib, the most common severe hematologic AE was neutropenia. The hematologic toxicities profile of niraparib differs from that of both olaparib and veliparib, thrombocytopenia and anemia were the most common hematologic AEs with niraparib, which occurred more commonly in the early cycles of therapy. Similarly, a Phase I trial also reported thrombocytopenia as one of the most common treatmentrelated toxic effects of niraparib. ${ }^{30}$ What is noteworthy is that severe thrombocytopenia was common with niraparib, but the incidence of discontinuation is low due to such events by dose modifications and delays. ${ }^{9}$ Although the experience of severe hematologic toxicities is one of the main toxicity challenges of PARPis treatment, there are currently no methods to predict patients at higher risk, therefore regular monitoring of complete blood counts is recommended. The prescribing information of niraparib suggests that it should be used in patients who have recovered from hematological toxicity caused by previous chemotherapy. Complete blood counts should be monitored weekly for the first month, monthly for the next 11 months of treatment, and periodically after this time. If hematological toxicities do not resolve within 28 days following interruption, niraparib should be discontinued, and the patient should be referred to a hematologist for further investigations, including bone marrow analysis and blood sample for cytogenetics (www.accessdata.fda.gov/ drugsatfda docs/label/2017/2084471bl.pdf).

Challenges of combinations of PARPis and chemotherapy agents include an increased risk of developing myelosuppression. Oza et al reported that severe neutropenia was more common with olaparib plus chemotherapy than with chemotherapy, which suggested that concurrent administration of a PARPi with platinum-based chemotherapy might intensify platinum-induced neutropenia. ${ }^{10}$ Our study showed that the addition of PARPis to single-agent chemotherapy was associated with a significantly increased risk of severe hematologic toxicities. Interestingly, PARPis combined with carboplatin and paclitaxel was not associated with increased risk of severe neutropenia, thrombocytopenia, and anemia in this analysis. However, this finding is not surprising, as carboplatin and paclitaxel utilized in both arms of these trials, likely drove the severity of the cytopenias, thereby diminishing the difference in severity of cytopenias among the treatment arms. Although myelosuppression is being increasingly recognized in PARPis combination trials with chemotherapy, the mechanism is unclear at this time. PARPis have several known mechanisms of action including inhibition of DNA SSB repair by BER leading to doublestrand breaks; alteration of nonhomologous recombination (HR) DNA repair pathways, such as promotion of classic non-homologous end joining, a very error-prone process of repair, which can lead to genetic instability; and disruption of alternative end joining which is essential for HR-deficient cells. PARPis prevent dissociation of recruited PARPs from DNA-damage sites, and these stabilized PARP/DNA complexes are lethal to HR-deficient cells. ${ }^{31}$ Chemotherapy agents can potentiate DNA damage, and PARPis diminish the PARP enzymes' ability to repair DNA damage. Since PARP inhibition is not selective for tumor cells, inhibition of PARP in normal cells abrogates an important mechanism of DNA repair in these cells, thereby enhancing toxicity from chemotherapy, including myelosuppression.

In order to reduce the risk of hematologic toxicities, the sequence of agents, drug dosing choices, and potential for drug interactions in humans should be considered. An intermittent schedule of PARPis administration instead of continuous dosing has proved effective in reducing toxicity. ${ }^{32}$ Lee et al showed that carboplatin pre-exposure caused intracellular olaparib accumulation reducing bioavailable olaparib, predicting carboplatin should be administered prior to olaparib. ${ }^{33}$ CYP3A inhibitors and inducers should not be used concomitantly with olaparib, as olaparib is primarily metabolized by the CYP3A enzymes, and coadministration with CYP3A inhibitors or inducers may affect metabolic clearance and alter plasma concentration of the drug. ${ }^{34}$ Niraparib may induce CYP1A2 and have the potential to interact with CYP metabolism, so, concomitant use with drugs that are metabolized by CYP1A2 is not 
recommended. Moreover, niraparib clearance can also be affected by concurrent administration of P-gp inhibitors or substrates. ${ }^{9}$ Furthermore, since thrombocytopenia is common with niraparib and talazoparib, caution should be exercised when these agents are coadministered with antiplatelet and anticoagulation drugs. ${ }^{9,35}$

However, there are several limitations that need to be considered in our meta-analysis. First, this is a meta-analysis at study level, not on the patients' data, therefore confounding variables at the patient level, such as previous treatment exposure and concomitant treatments, cannot be assessed properly and incorporated into the analysis. Second, the reported incidence and RRs of hematologic AEs have significant heterogeneity among the included studies. And our subgroup analysis showed that different PARPis included in the meta-analysis might partly explain the heterogeneity. Moreover, different concomitant therapies (placebo or single-agent chemotherapy or combination chemotherapy), dosage of PARPis, and types of malignancies might be another source of heterogeneity. We used random effects model to minimize the influence of heterogeneity. Third, evidence of publication bias was observed for the RRs of severe neutropenia by both the Egger and the Begg test. This might be related to the inclusion of small studies and the between-trial heterogeneity. Fourth, the number of trials was limited for some subgroup analyses, eg, only one RCT evaluated niraparib, and this is also the single study evaluated monotherapy and placebo group when calculating the risk of severe neutropenia and thrombocytopenia. As the RRs for niraparib are exceptionally high when compared with other PARPis, the results of these subgroup analysis might be affected by this RCT. Finally, as no published RCTs with rucaparib were found, our analysis did not include rucaparib for the calculation of incidence and RRs of hematologic toxicities.

\section{Conclusion}

In conclusion, this meta-analysis of RCTs demonstrates that PARPis treatment is associated with an increased risk of developing severe hematologic toxicities. In subgroup analysis, the risk of hematologic toxicities may vary with drug types and concomitant use of anticancer agents. In order to improve outcome and quality of life of patients, clinicians should be aware of these risks and perform regular hematologic monitoring.

\section{Disclosure}

The authors report no conflicts of interest in this work.

\section{References}

1. de Murcia G, Ménissier-de Murcia J, Schreiber V. Poly(ADP-ribose) polymerase: molecular biological aspects. Bioessays. 1991;13(9): 455-462.

2. Sousa FG, Matuo R, Soares DG, Escargueil AE, Henriques JA, Larsen AK, Saffi J. PARPs and the DNA damage response. Carcinogenesis. 2012;33(8):1433-1440.

3. Ashworth A. A synthetic lethal therapeutic approach: poly(ADP) ribose polymerase inhibitors for the treatment of cancers deficient in DNA double-strand break repair. J Clin Oncol. 2008;26(22):3785-3790.

4. Farmer $\mathrm{H}, \mathrm{McCabe} \mathrm{N}$, Lord CJ, et al. Targeting the DNA repair defect in BRCA mutant cells as a therapeutic strategy. Nature. 2005; 434(7035):917-921.

5. Kim G, Ison G, McKee AE, et al. FDA approval summary: olaparib monotherapy in patients with deleterious germline BRCA-mutated advanced ovarian cancer treated with three or more lines of chemotherapy. Clin Cancer Res. 2015;21(19):4257-4261.

6. Syed YY. Rucaparib: first global approval. Drugs. 2017;77(5): 585-592.

7. Scott LJ. Niraparib: first global approval. Drugs. 2017;77(9): 1029-1034.

8. Konecny GE, Kristeleit RS. PARP inhibitors for BRCA1/2-mutated and sporadic ovarian cancer: current practice and future directions. Br J Cancer. 2016;115(10):1157-1173.

9. Mirza MR, Monk BJ, Herrstedt J, et al. Niraparib maintenance therapy in platinum-sensitive, recurrent ovarian cancer. $N$ Engl J Med. 2016;375(22):2154-2164.

10. Oza AM, Cibula D, Benzaquen AO, et al. Olaparib combined with chemotherapy for recurrent platinum-sensitive ovarian cancer: a randomised phase 2 trial. Lancet Oncol. 2015;16(1):87-97.

11. Middleton MR, Friedlander P, Hamid O, et al. Randomized phase II study evaluating veliparib (ABT-888) with temozolomide in patients with metastatic melanoma. Ann Oncol. 2015;26(10):2173-2179.

12. Moher D, Liberati A, Tetzlaff J, Altman DG; PRISMA Group. Preferred reporting items for systematic reviews and meta-analyses: the PRISMA statement. Int J Surg. 2010;8(5):336-341.

13. Jadad AR, Moore RA, Carroll D, Jenkinson C, Reynolds DJ, Gavaghan DJ, McQuay HJ. Assessing the quality of reports of randomized clinical trials: is blinding necessary? Control Clin Trials. 1996;17(1): $1-12$.

14. DerSimonian R, Laird N. Meta-analysis in clinical trials. Control Clin Trials. 1986;7(3):177-188.

15. Lau J, Ioannidis JP, Schmid CH. Quantitative synthesis in systematic reviews. Ann Intern Med. 1997;127(9):820-826.

16. Higgins JP, Thompson SG, Deeks JJ, Altman DG. Measuring inconsistency in meta-analyses. BMJ. 2003;327(7414):557-560.

17. Begg CB, Mazumdar M. Operating characteristics of a rank correlation test for publication bias. Biometrics. 1994;50(4):1088-1101.

18. Egger M, Davey Smith G, Schneider M, Minder C. Bias in meta-analysis detected by a simple, graphical test. BMJ. 1997;315(7109):629-634.

19. Bang YJ, Im SA, Lee KW, et al. Randomized, double-blind Phase II trial with prospective classification by ATM protein level to evaluate the efficacy and tolerability of olaparib plus paclitaxel in patients with recurrent or metastatic gastric cancer. J Clin Oncol. 2015;33(33):3858-3865.

20. Ledermann J, Harter P, Gourley C, et al. Olaparib maintenance therapy in platinum-sensitive relapsed ovarian cancer. $N$ Engl J Med. 2012;366(15):1382-1392.

21. Kummar S, Oza AM, Fleming GF, et al. Randomized trial of oral cyclophosphamide and veliparib in high-grade serous ovarian, primary peritoneal, or fallopian tube cancers, or BRCA-mutant ovarian cancer. Clin Cancer Res. 2015;21(7):1574-1582.

22. Kaye SB, Lubinski J, Matulonis U, et al. Phase II, open-label, randomized, multicenter study comparing the efficacy and safety of olaparib, a poly (ADP-ribose) polymerase inhibitor, and pegylated liposomal doxorubicin in patients with BRCA1 or BRCA2 mutations and recurrent ovarian cancer. J Clin Oncol. 2012;30(4):372-379. 
23. Chabot P, Hsia TC, Ryu JS, et al. Veliparib in combination with wholebrain radiation therapy for patients with brain metastases from non-small cell lung cancer: results of a randomized, global, placebo-controlled study. J Neurooncol. 2017;131(1):105-115.

24. Rugo HS, Olopade OI, DeMichele A, et al. Adaptive randomization of veliparib-carboplatin treatment in breast cancer. $N$ Engl J Med. 2016; 375(1):23-34.

25. Ramalingam SS, Blais N, Mazieres J, et al. Randomized, placebocontrolled, Phase II study of veliparib in combination with carboplatin and paclitaxel for advanced/metastatic non-small cell lung cancer. Clin Cancer Res. 2017;23(8):1937-1944.

26. Pietanza MC, Krug LM, Waqar SN, et al. A multi-center, randomized, double-blind phase II study comparing temozolomide (TMZ) plus either veliparib (ABT-888), a PARP inhibitor, or placebo as 2nd or 3rd-line therapy for patients (Pts) with relapsed small cell lung cancers (SCLCs). J Clin Oncol. 2016;34(15 suppl):8512.

27. Han HS, Diéras V, Robson ME, et al. Efficacy and tolerability of veliparib (V; ABT-888) in combination with carboplatin (C) and paclitaxel (P) vs placebo $(\mathrm{Plc})+\mathrm{C} / \mathrm{P}$ in patients (pts) with BRCA1 or BRCA2 mutations and metastatic breast cancer: a randomized, phase 2 study. Cancer Res. 2017;77(4 Suppl):abstract S2-05.

28. Fong PC, Boss DS, Yap TA, et al. Inhibition of poly(ADP-ribose) polymerase in tumors from BRCA mutation carriers. $N$ Engl J Med. 2009; 361(2):123-134

29. Tutt A, Robson M, Garber JE, et al. Oral poly(ADP-ribose) polymerase inhibitor olaparib in patients with BRCA1 or BRCA2 mutations and advanced breast cancer: a proof-of-concept trial. Lancet. 2010; 376(9737):235-244
30. Sandhu SK, Schelman WR, Wilding G, et al. The poly(ADP-ribose) polymerase inhibitor niraparib (MK4827) in BRCA mutation carriers and patients with sporadic cancer: a phase 1 dose-escalation trial. Lancet Oncol. 2013;14(9):882-892.

31. Konstantinopoulos PA, Ceccaldi R, Shapiro GI, D’Andrea AD. Homologous recombination deficiency: exploiting the fundamental vulnerability of ovarian cancer. Cancer Discov. 2015;5(11):1137-1154.

32. Balmaña J, Tung NM, Isakoff SJ, et al. Phase I trial of olaparib in combination with cisplatin for the treatment of patients with advanced breast, ovarian and other solid tumors. Ann Oncol. 2014;25(8): $1656-1663$.

33. Lee JM, Peer CJ, Yu M, et al. Sequence-specific pharmacokinetic and pharmacodynamic Phase I/Ib study of olaparib tablets and carboplatin in women's cancer. Clin Cancer Res. 2017;23(6):1397-1406.

34. Dirix L, Swaisland H, Verheul HM, et al. Effect of itraconazole and rifampin on the pharmacokinetics of olaparib in patients with advanced solid tumors: results of two Phase I open-label studies. Clin Ther. 2016;38(10):2286-2299.

35. De Bono JS, Mina LA, Gonzalez M, et al. First-in-human trial of novel oral PARP inhibitor BMN 673 in patients with solid tumors. J Clin Oncol. 2013;31(15 suppl):2580-2580.

\section{Publish your work in this journal}

Drug Design, Development and Therapy is an international, peerreviewed open-access journal that spans the spectrum of drug design and development through to clinical applications. Clinical outcomes, patient safety, and programs for the development and effective, safe, and sustained use of medicines are the features of the journal, which

\section{Dovepress}

has also been accepted for indexing on PubMed Central. The manuscript management system is completely online and includes a very quick and fair peer-review system, which is all easy to use. Visit http://www.dovepress.com/testimonials.php to read real quotes from published authors.

Submit your manuscript here: http://www.dovepress.com/drug-design-development-and-therapy-journal 